\title{
ESTRATEGIAS DE DECISIÓN EN SISTEMAS DINÁMICOS: APLICANDO MAPAS COGNITIVOS DIFUSOS APLICACIÓN A UN EJEMPLO SOCIO - ECONÓMICO
}

\author{
DECISION STRATEGIES IN DYNAMIC SYSTEMS USING FUZZY \\ COGNITIVE MAPS. APPLICATION TO A SOCIO - ECONOMIC \\ EXAMPLE
}

\author{
Lisandro Curia \\ Andrea Lavalle \\ Universidad Nacional del Comahue, Buenos Aires, Argentina
}

\begin{abstract}
Situations in which human beings develop their daily tasks are extremely complex and dynamic. In each field, the analysis of the system`s variables under consideration can be simplified if the system is conceived as a set of concepts, where a change in each of them will cause changes in the remainder. In the analysis of a particular problem, the representation of concepts in the form of a map helps to synthesize information detecting the main concepts that are linked to the problem. In these cases, Fuzzy Cognitive Maps (FCM) are able to synthesize much of this information. Also with this technique is possible to follow the evolution of the concepts to a state of equilibrium and therefore allow us to study the dynamics that takes the passage from one state to another given the situation under review. This paper describes the construction and analysis of the Fuzzy Cognitive Maps technique through an economy application example. The aim is to present a methodology supported by the FCM to analyze the evolution and impact on the system causing by the change in the value of one or more concepts involved.
\end{abstract}

Keywords: Fuzzy logic, dynamic cualitative systems, cognitive maps.

Manuscript first received/Recebido em 05/03/2011 Manuscript accepted/Aprovado em: 17/11/2011

Address for correspondence / Endereço para correspondência

Leopoldo Lisandro Horacio Curia, Ingeniero Mecánico por la Universidad Tecnológica Nacional Regional Santa Fe. Magíster en Enseñanza de las Ciencias Exactas y Naturales, orientación Matemática por la Universidad Nacional del Comahue. Profesor Adjunto Regular del Departamento de Matemática de la Universidad Nacional del Comahue. Dpto. de Matemática Facultad de Economía y Administración. Universidad Nacional del Comahue. Buenos Aires 1400, (8300) Neuquén. Argentina. +54-299-4490300. lcuria@uncoma.edu.ar

Andrea Lina Lavalle, Profesora en Matemática por la Universidad Nacional del Comahue. Magíster en Enseñanza de las Ciencias Exactas y Naturales, orientación Matemática por la Universidad Nacional del Comahue. Profesora Adjunta Interina del Departamento de Estadística de la Universidad Nacional del Comahue. Dpto. de Estadística. Facultad de Economía y Administración. Universidad Nacional del Comahue. Buenos Aires 1400, (8300) Neuquén. Argentina. +54-299-4490300. lavalleandrea@yahoo.com.ar 


\section{RESUMEN}

Las situaciones que abarcan el contexto en que el ser humano desarrolla sus tareas cotidianas son extremadamente complejas y dinámicas. En cada campo, el análisis de las variables que componen el sistema objeto de estudio puede simplificarse si se lo concibe compuesto como un conjunto de conceptos donde un cambio en cada uno de ellos provocará cambios en los restantes. Cuando se trata de analizar la problemática de un área particular, la representación de los conceptos en forma de un mapa permite sintetizar la información aislando los principales conceptos que están vinculados en el problema. En estos casos los Mapas Cognitivos Difusos (MCD) logran sintetizar gran parte de la información presente. Además con esta técnica es posible seguir la evolución de los conceptos hasta un estado de equilibrio y por lo tanto permiten estudiar la dinámica que lleva el pasaje de un estado a otro determinado en la situación que se analiza. En este trabajo se expone la técnica de construcción y análisis de Mapas Cognitivos Difusos a través de un ejemplo de aplicación a la economía. El objetivo consiste en presentar una metodología de trabajo apoyada en los MCD que permite analizar la evolución y el impacto que provoca en el sistema el cambio en el valor de uno o varios conceptos intervinientes.

Palabras clave: Lógica difusa, sistemas cualitativos dinámicos, mapas cognitivos

\section{INTRODUCCIÓN}

La toma de decisiones en ámbitos como la política y la educación pasando por la economía y la salud, constituye un serio desafío. Normalmente, establecer prioridades y pronosticar determinadas situaciones puede no ser una tarea sencilla aún disponiendo de personal idóneo y con conocimiento y experiencia suficiente como para llevar adelante esa tarea (Carlsson, 1996; Wheeldon \& Faubert, 2009). La propia dinámica de los acontecimientos y el número de variables que componen el sistema objeto de estudio añaden una complejidad adicional a la situación que se quiere analizar. Desde el punto de vista estadístico y computacional, modelar un sistema que evoluciona en el tiempo (sistema dinámico) es una tarea compleja; por ejemplo, los modelos matemáticos para analizar el comportamiento de este tipo de sistemas se desarrollan en base a ecuaciones diferenciales o mediante sistemas de ecuaciones diferenciales (Jang \& Mitsutani, 1997). Aún así, en estos modelos determinísticos debe conocerse completamente la expresión matemática que liga las variables que componen el sistema. Este es un hecho poco común en muchos sistemas o situaciones en las que el ser humano desarrolla sus actividades. Si bien existen modelos matemáticos que describen fenómenos físicos, sistemas mecánicos, velocidades de reacción, transmisión de calor, etc., existen otras situaciones, principalmente aquellas en las que interviene el factor humano, donde es imposible establecer un modelo matemático.

En estos ambientes donde el ser humano interviene es frecuente calificar situaciones y reacciones con expresiones lingüísticas y no numéricas. Surgen así sistemas donde se describe la realidad en términos cualitativos y por ello se denominan sistemas cualitativos (Carvalho \& Tomé, 1999). De este modo, se entiende que los sistemas cualitativos son difíciles o imposibles de ser descriptos con leyes matemáticas y están rodeados de factores como incertidumbre e imprecisión. Estos sistemas abarcan una amplia gama de situaciones que van desde el comportamiento y actitud individual que puede exhibir, por ejemplo, el presidente de una compañía que elabora un producto, hasta conductas más generalizadas presentes en agrupamientos sectoriales de una sociedad que engloba un determinado número de personas, y que puede llegar a generar conflictividad social, procesos inflacionarios, crecimiento económico, inestabilidad en el gobierno, etc. (Lin et al., 2010; Stylios et al., 2005). Otros conceptos sustentados por la sociedad como poder adquisitivo, riqueza, pobreza, libertad, bienestar, etc., son también ejemplos de conceptos que componen sistemas cualitativos. Como puede verse, 
los sistemas socio-económicos son sistemas cualitativos por excelencia y en realidad, debido a los continuos cambios que se suceden en estos ámbitos, corresponde hablar de sistemas cualitativos dinámicos.

\section{Planteo del problema}

El problema de investigación que se aborda en el presente trabajo se relaciona con la necesidad de contar con herramientas que, mediante un tratamiento cualitativo y cuantitativo, permitan predecir el comportamiento que tendrán las componentes de un sistema cualitativo dinámico. Como puede observarse, el problema es de tipo metodológico y para dar respuesta al mismo se propone la utilización combinada de un mapa cognitivo con técnicas de la lógica difusa ya que aportan elementos que permiten traducir expresiones verbales y dichos ambiguos en valores numéricos concretos. Se analiza un caso concreto de aplicación a la economía aunque la metodología propuesta puede extenderse con facilidad a otras áreas relacionadas con la toma de decisiones y estrategias políticas y de producción.

\section{Objetivos}

- Presentar una metodología de trabajo apoyada en los Mapas Cognitivos Difusos que permite analizar la evolución de un sistema cualitativo dinámico

- Ilustrar la técnica de construcción y análisis de Mapas Cognitivos Difusos a través de un ejemplo de aplicación a una problemática socio-económica.

Este trabajo está compuesto de cuatro secciones. En la primera se establecen los lineamientos teóricos que dan sustento a la investigación, se ejemplifican los sistemas cualitativos y se definen diferentes tipos de mapas: conceptuales, cognitivos y cognitivos difusos, como formas de representación del conocimiento. En la segunda sección se describe la propuesta metodológica donde se recurre a un ejemplo concreto para mostrar cómo se construye y se procesa un mapa cognitivo difuso. En la tercera sección se muestran los resultados de la implementación de un mapa cognitivo difuso aplicado a un problema de toma de decisiones. En la última sección se detallan las conclusiones del trabajo y las líneas futuras de investigación.

\section{MARCO TEÓRICO}

Antes de pasar a describir la técnica que involucra la implementación y el análisis de un Mapa Cognitivo Difuso se presentará brevemente una situación que pone de manifiesto cómo los conceptos que intervienen en un sistema cualitativo se ven afectados por los cambios que puedan presentar uno o varios de los conceptos restantes.

Considérese por ejemplo, un pasajero que debe viajar por una autopista. Los conceptos a incluir para conformar el sistema pueden ser: mal tiempo, aversión a conducir, congestión de tránsito, estado de la autopista, estado del automóvil, velocidad a la que se conduce, entre otras variables que puedan tener relevancia en el sistema. Las variables del sistema generalmente son seleccionadas por uno o varios especialistas que van a analizar la problemática determinada. Estas variables pasarán a ser luego los conceptos del mapa cognitivo. Por ejemplo, otro especialista perteneciente al área en el que se está analizando el problema del tránsito podría incluir como variables la frecuencia de control de la policía, día de la semana, etc. Los conceptos se influyen mutuamente (Papageorgiou et al., 2006; Miao et al., 2006) y esta influencia puede ser positiva o negativa y su intensidad puede ser alta o baja. Por ejemplo, el mal tiempo 
conduce a un aumento del congestionamiento de tránsito y determina una disminución de la velocidad. Si la frecuencia del control policial aumenta, el número de accidentes baja. El número de accidentes no afecta directamente la propia velocidad del que conduce, pero si este número aumenta, el propio sentido de riesgo del conductor condiciona su aversión a conducir y como la aversión aumenta, el conductor tiende a retrasarse. Estos escenarios y otros similares donde se describen situaciones económicas, políticas y sociales pueden representarse gráficamente por medio de conceptos en un papel y luego implementarse en una computadora a través de los denominados Mapas Cognitivos donde los conceptos reciben el nombre de nodos y las aristas que conectan los conceptos se denominan eventos causales (Calais, 2008; Kosko, 1986).

Existen diversos tipos de mapas que pueden utilizarse para representar información. Los mapas conceptuales permiten la organización jerárquica del conocimiento de un tema y pueden utilizarse para analizar y sintetizar información (Novak \& Gowin, 1988). Un mapa conceptual representa una jerarquía de diferentes niveles de generalidad e inclusividad conceptual y se compone de conceptos, proposiciones y palabras enlace. Los conceptos se refieren a objetos, eventos o situaciones y se representan en círculos. Existen tres tipos de conceptos: supraordinados (mayor nivel de inclusividad), coordinados (igual nivel de inclusividad) y subordinados (menor nivel de inclusividad). Las proposiciones representan la unión de dos o más conceptos relacionados entre sí mediante una palabra enlace. Las palabras enlace, expresan el tipo de relación existente entre dos o más conceptos y se representan a través de líneas rotuladas (Carvalho \& Tomé, 1999; Mellado et al., 2002). Las técnicas de construcción de mapas conceptuales son muy utilizadas para la enseñanza en los diferentes niveles educativos.

Los mapas cognitivos permiten analizar contenidos temáticos de una disciplina, situaciones personales o grupales y también los procesos que intervienen en la formación de estrategias operativas. Asimismo, proporcionan una imagen mental personal tomada del ambiente y a la que puede recurrirse para analizar informalmente los cambios que se producen en el medio que se está analizando cuando se modifican los estímulos ambientales originales (Carlsson, 1996; Axelrod, 1976).

Los mapas cognitivos relacionan de una forma parcialmente jerarquizada, unidades de información con un sentido más amplio que los mapas conceptuales tradicionales de Novak. Aunque tanto la representación de conceptos por medio de mapas cognitivos o a través de un mapa conceptual, permiten una visión global y no fragmentada de las concepciones que cada experto tiene del área de estudio, los primeros han sido utilizados mayormente en estrategias de planeamiento y los mapas conceptuales se utilizan principalmente en educación y en el estudio del comportamiento humano, pero ambos atienden a situaciones estáticas, dejando de lado las componentes dinámicas que implican la evolución y posterior desenvolvimiento de una situación (Kardaras \& Karakostas, 1999). Es por ello que los mapas cognitivos se utilizan en un marco donde la dinámica del sistema y la jerarquización de los conceptos se dejan de lado.

Los mapas cognitivos admiten diferentes formas de representación: tipo sol, de nubes, de ciclos y de secuencias, entre otras. Por ejemplo, en la representación tipo sol, el concepto central se representa con un círculo en el medio del esquema y el resto de los conceptos se muestran con varios rayos dirigidos hacia este centro que dan a entender cómo influyen las ideas representadas en los rayos sobre el concepto central. 
En el mapa cognitivo en forma de nubes, se coloca en la nube central el tema principal y alrededor de ella se colocan otras nubes que contienen subtemas. Estas llevan información adicional que se desea aportar. Un mapa cognitivo muy utilizado es el denominado de satélites. En la parte central se coloca el tema de interés o el concepto principal enmarcado en un círculo y en rectángulos periféricos se distribuyen los subtemas simulando satélites que giran en torno al tema central. De los rectángulos salen flechas que indican la incidencia de los subtemas en el tema central (Pimienta, 2005).

Los mapas cognitivos difusos (MCD) constituyen una herramienta desarrollada por Kosko (1986) que tiene por finalidad expandir el horizonte de trabajo que supone la implementación de los mapas cognitivos tradicionales y los mapas conceptuales de Novak (Carlsson et al., 2006; Kosko, 1997). Estos mapas, en versiones más avanzadas, pueden incluir la combinación de técnicas pertenecientes a la Soft Computing, tales como las Redes Neuronales Artificiales y la Lógica Difusa (Jang et al., 1997; Kosko, 1997). Si bien el método de construcción de un MCD no puede considerarse una técnica infalible, puesto que algunos conceptos pueden dejarse de lado y finalmente su ausencia podría ser un factor crítico en el análisis final de la estrategia a seguir, constituyen una herramienta de gestión muy útil y de gran impacto visual a la hora de representar y resumir la información (Cai et al., 2006; Chandana et al., 2007). Aun así, los MCD pertenecen a un área en constante expansión y con un campo de aplicación cada vez mayor, donde actualmente se investigan alternativas de mejora, tal es el caso de los MCD con base de reglas borrosas (BR-MCD) (Carvalho \& Tomé, 1999) cuya construcción se basa en la aplicación de reglas difusas un tanto más complejas que las detalladas en este trabajo. Estos sistemas muestran ser un excelente complemento a los MCD tradicionales para estudiar la evolución de los conceptos en aquellos casos en los casos en que los MCD tradicionales no presenten buenas características de convergencia o cuando se quiera representar el conocimiento impreciso o el lenguaje ambiguo de los especialistas con una base de reglas operativas llamadas reglas borrosas (Carvalho \& Tomé, 1999). Este aspecto es un elemento a tener en cuenta a la hora de modelar sistemas en los que intervienen los seres humanos ya que el modo de comunicar sus ideas sobre hechos, situaciones o acontecimientos determinados siempre contiene información expresada con ambigüedad e imprecisión lo que dificulta o imposibilita disponer de modelos que trabajen con valores numéricos concretos.

Formalmente, un mapa cognitivo difuso consiste de un grafo dirigido con varios nodos que representan los conceptos causales que surgen del tema a tratar y de arcos dirigidos conectados a los nodos que representan las relaciones causales entre los mismos (Stach et al., 2010). Inicialmente cada concepto toma valores 0 ó 1 , el valor 0 indica la ausencia del concepto en el instante en que se analiza el problema mientras que valor 1 indica la presencia del concepto. Existen otros MCD donde los nodos, llamados nodos difusos, toman valores pertenecientes al intervalo $[0,1]$.

Las aristas que conectan los nodos tienen asociado un peso cuyo valor puede pertenecer al conjunto $\{-1,0,1\}$ o al intervalo $[-1,1]$. Estos valores indican el peso o la intensidad con que un concepto influye en el otro. El peso será positivo si representa una relación causal incremental y negativo si la conexión implica una relación causal decreciente (Carvalho \& Tomé, 1999). El término difuso proviene del hecho con que estos valores son asignados por uno o varios expertos y por tanto conllevan algún grado de información cualitativa propia de la interpretación del experto y que es introducida al mapa como un valor concreto. 
Con la finalidad de ilustrar los tres tipos de mapas enunciados, se presenta un ejemplo tomado del texto de Kandasamy y Smarandache (2003) en el que, en un escenario de una dinámica compleja de mercado, se establecen los factores que influyen en el mismo clasificados como factores incontrolables, controlables y semicontrolables. Sobre los mismos conceptos se realizan tres representaciones: un mapa conceptual, un mapa cognitivo y un mapa cognitivo difuso.

En el caso del mapa conceptual representado en la Figura 1, se parte del concepto más inclusivo, que es el mercado, y se han puesto en un mismo nivel jerárquico los tres tipos de factores, sin embargo puede resultar de interés distinguir los órdenes jerárquicos de los ejemplos de cada uno de los tres casos. Así en una situación especial de mercado, para el que diseña el mapa conceptual puede ser de mayor importancia incluir el concepto "demanda" en un nivel jerárquico más alto porque por ejemplo podría hacer referencia a una situación especial de alta demanda del producto por condiciones específicas del mercado.

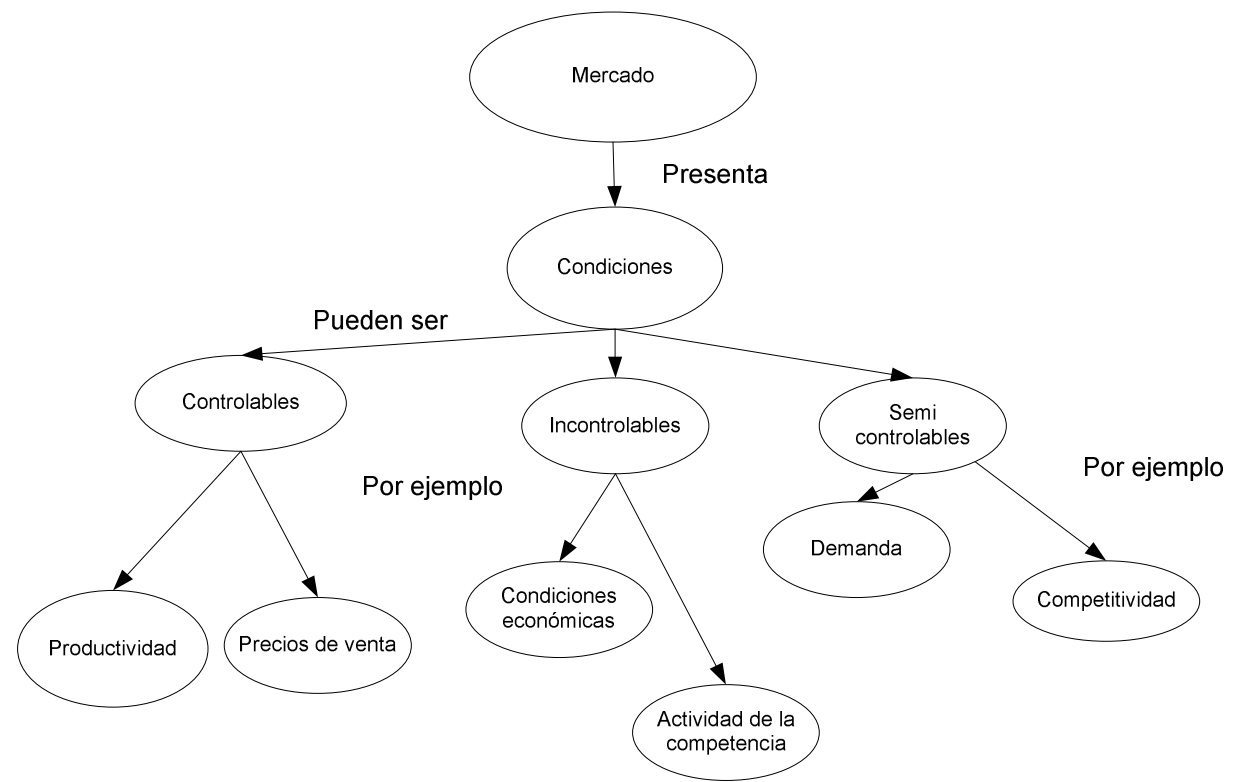

Figura 1: Representación de conceptos en un Mapa Conceptual

En la Figura 2 se representa la misma problemática a través de un mapa cognitivo. En este caso los conceptos no están ordenados jerárquicamente y hay libertad para disponerlos en distintos sectores del mapa. El mapa cognitivo es del tipo satélite y contiene una idea central que es el mercado y los temas secundarios están distribuidos en rectángulos con flechas orientadas hacia la idea central. 


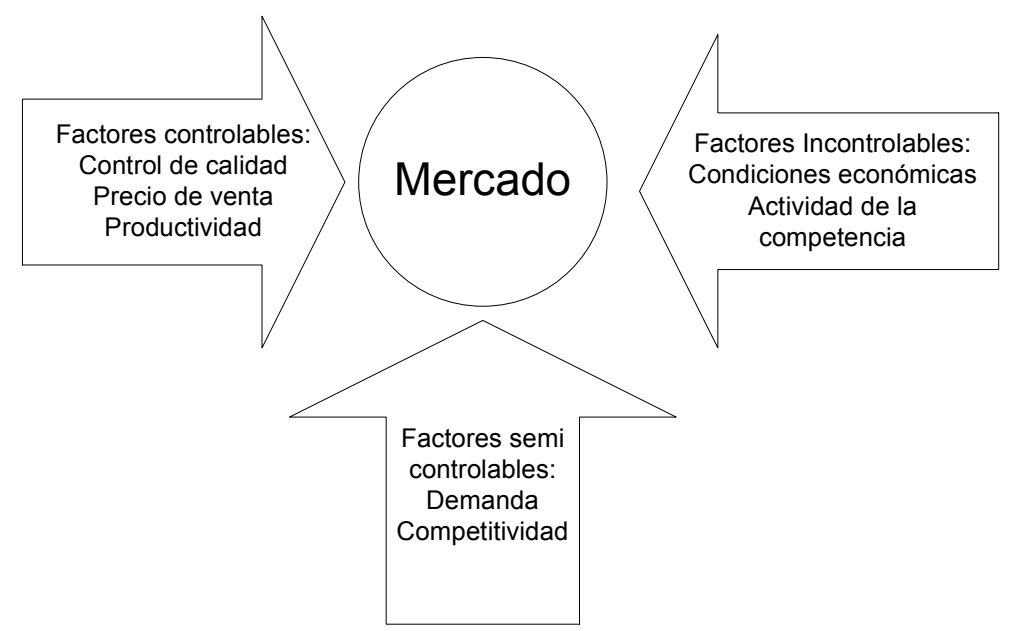

Figura 2: Conceptos en un Mapa Cognitivo

En la comparación entre un mapa conceptual y un mapa cognitivo debe mencionarse un aspecto de fundamental importancia que los distingue. El mapa conceptual presenta una estructura lógica aceptada socialmente por los expertos del tema. En cambio el mapa cognitivo tiene una estructura con un sesgo hacia el campo de la psicología y pretende formar una representación idiosincrásica personal (Axelrod, 1976).

Finalmente en la Figura 3 se utiliza un Mapa Cognitivo Difuso. Aquí los conceptos están relacionados con flechas que tienen adicionados un signo más o un signo menos de acuerdo al caso.

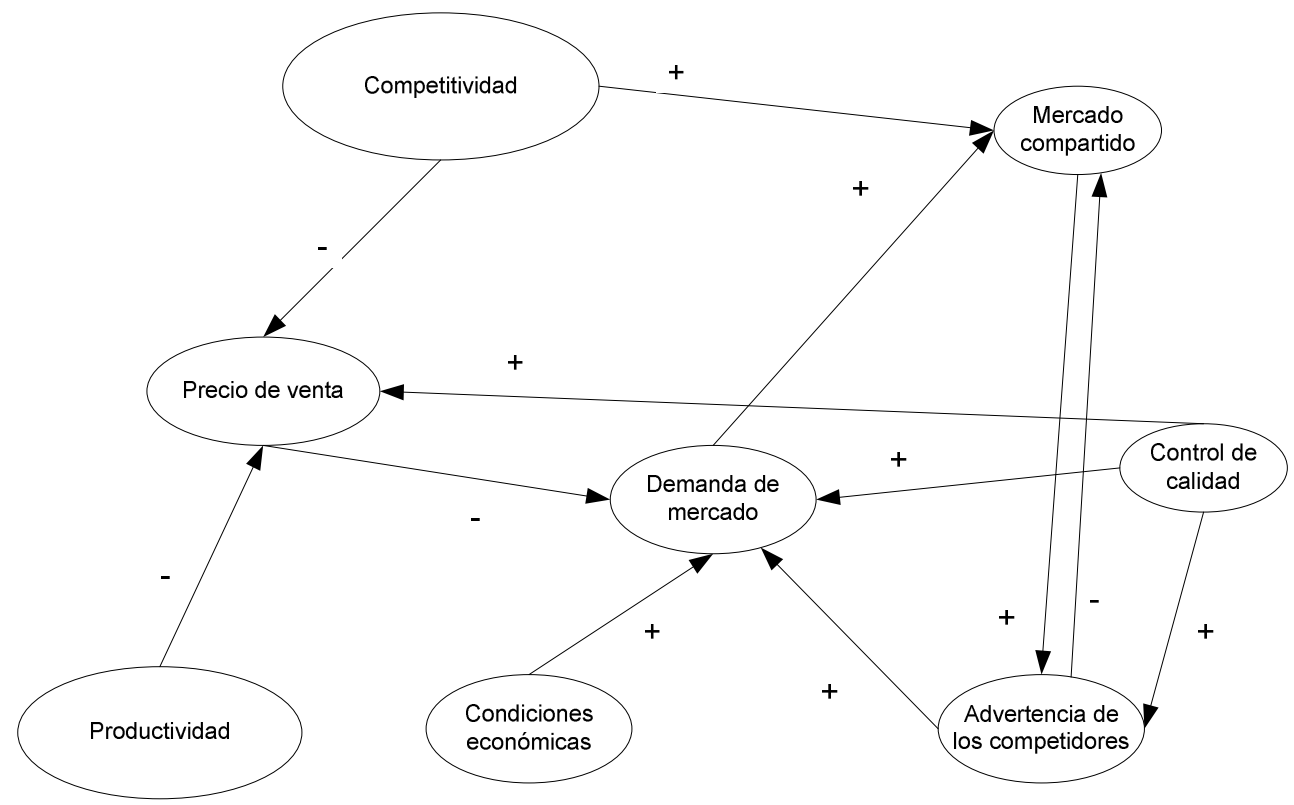

Figura 3: Conceptos en un Mapa Cognitivo Difuso

Para este ejemplo particular, el experto que evalúa la incidencia que tienen entre sí los conceptos considera, por ejemplo, que el control de calidad tiene una incidencia positiva en la demanda de mercado y el precio de venta una incidencia negativa sobre el mismo.

Lo que se pretende lograr con el estudio de un MCD es básicamente obtener la respuesta a un cuestionamiento de la forma "que ocurre si". Por ello dentro de un 
conjunto de conceptos reunidos se pretende analizar qué le sucede al sistema si algún estímulo cambia el valor de los conceptos, o bien si se introducen nuevos conceptos o se quitan algunos existentes. Este análisis en la dinámica del sistema, puede lograrse mediante un MCD que operando de manera iterativa, de modo de ir actualizando los valores de los conceptos secuencialmente y luego de cierto número de iteraciones, llegue a un nuevo estado de equilibrio que permita concluir qué consecuencias trajeron aparejadas los cambios realizados en los valores de los conceptos (Hilera \& Martínez, 2000). La estrategia a seguir consiste en comenzar con un cierto valor inicial de los conceptos y manteniendo fijos los valores de las conexiones entre ellos, analizar el cambio que opera en los valores de los mismos de acuerdo con la dinámica propia del sistema.

Las posibilidades de aplicación de los mapas cognitivos difusos se están extendiendo en la actualidad a diversos campos disciplinares y en diferentes ámbitos de la toma de decisiones. En el trabajo de Stach et al. (2010) se resalta el amplio uso de los mapas cognitivos difusos debido en gran medida a su sencillez y facilidad de uso. Estos autores resaltan los avances que ha habido en los últimos años en la construcción prácticamente automática de estos mapas. Sin embargo, cuando el número de conceptos que contiene el mapa es elevado su construcción se torna compleja, inclusive si el desarrollo del mapa recae sobre un experto. Por tal motivo, los autores proponen recurrir a otra rama de la inteligencia artificial como son los algoritmos genéticos con objeto de depurar las variables que contiene el problema. Los algoritmos genéticos fueron ampliamente utilizados en problemas de optimización y reducción de variables. En este caso los autores presentan el método de construcción de un mapa cognitivo difuso partiendo de un gran número de mapas y optimizando las variables que intervienen en cada subconjunto hasta quedarse con un modelo definitivo. Estos trabajos, basados en rutinas de optimización con base en los algoritmos genéticos, están ocupando un sector importante en la divulgación científica de la actualidad. Los algoritmos genéticos junto con las redes neuronales artificiales y la lógica difusa constituyen las bases de la denominada soft computing y a su vez son una rama importante de la inteligencia artificial la que cuenta con un enorme desarrollo tanto teórico como en la práctica. El empleo de estas rutinas de optimización en la construcción de un mapa cognitivo difuso sólo se justifican en estos casos, donde hay un gran número de conceptos. Si bien la inclusión de algoritmos genéticos en su forma netamente práctica no exige grandes complicaciones y existe software específico para su implementación, cuando el número de variables no es elevado no se justifica su empleo. Con un número reducido de conceptos los mapas cognitivos difusos ya han mostrado dar buenos resultados y su novedad consiste no tanto en optimizar su funcionamiento sino en dar una respuesta concreta, ágil y flexible a un problema de aplicación específico.

En medicina, los mapas cognitivos difusos también lograron encontrar su lugar ya que pueden colaborar en la toma de decisiones en diagnósticos médicos. Kannappan et al. (2011) proponen el empleo de mapas cognitivos difusos para modelar y predecir conductas en niños autistas. Su trabajo descansa en la combinación de modelos de redes neuronales con las técnicas clásicas de construcción de mapas cognitivos difusos. La importancia de su trabajo radica más en la utilidad que prestan los mapas cognitivos difusos en la predicción de trastornos en niños con capacidades especiales que en la efectividad de los mapas en sí. 
Por otro lado, en el trabajo de Papageorgiou et al. (2011) se realiza un estudio de la predicción del rendimiento de cultivos de algodón. Su estrategia vuelve a ser el empleo de la lógica difusa para modelar mediante una base de reglas difusas el conocimiento del experto en la temática. Ellos presentan un enfoque clásico del uso de los mapas cognitivos difusos también similar al que se emplea en este trabajo. Utilizan como nodos la textura del algodón, su $\mathrm{pH}$, materia orgánica, la producción de algodón y otros elementos químicos que forman parte del algodón. Su objetivo es presentar una herramienta que sea útil para predecir el rendimiento del algodón. Este trabajo pertenece a un área específica de la producción y está destinado concretamente a la mejora en la gestión de un cultivo.

Si bien en sus comienzos los mapas cognitivos difusos surgieron con el objetivo de ayudar a resolver problemas de estrategias políticas y de mercado, posteriormente, tal como muestran los trabajos citados, su empleo fue ramificándose hasta cubrir un gran número de aplicaciones aunque siempre relacionadas con estrategias de planeamiento y ayuda en la toma de dediciones.

\section{MATERIALES Y MÉTODOS}

\section{Técnicas para la construcción de Mapas Cognitivos Difusos}

Muchos fenómenos del mundo real pueden ser complicados si se los quiere representar por medio de un modelo matemático. En ocasiones para poder comprender la distribución de los principales conceptos y las relaciones que los vinculan, resulta de utilidad incorporar mapas cognitivos (Carlsson, 1996; Kosko, 1986; Kosko, 1997; Peláez \& Bowles, 1995). Cuando se requiera analizar la evolución de uno o varios conceptos intervinientes en el mapa al sufrir éste variaciones o cambios, resulta de interés estudiar el proceso dinámico que permita interpretar el estado al que evolucionó el sistema y las consecuencias que el estado inicial provocó. Pero no sólo captar la dinámica del sistema puede resultar complicado, un problema central que se plantea en la construcción de un sistema que permita representar conocimientos es el de atribuirle algún valor a la intensidad o al grado de influencia con la que un concepto provoca efectos o causalidades en otros. En general, esta relación viene expresada de manera ambigua pudiendo interpretarse de distintas formas como resultado natural de expresiones lingüísticas. Es completamente natural que sobre un mismo fenómeno varios especialistas tengan conocimientos y fuentes de valoraciones distintas. Con el fin de obtener una única valoración para la relación causal entre los conceptos se utilizan reglas de la lógica difusa. Más adelante se analizará esta situación con un caso concreto.

Si se está interesado en modelar un fenómeno o evento determinado que puede provenir del campo de la economía, relaciones sociales, salud, educación, etc., en primer lugar deben elegirse los conceptos que intervienen en la descripción del modelo y establecer las relaciones entre ellas. De acuerdo a Peláez (1995) el proceso de construcción del mapa cognitivo difuso de un sistema puede ser dividido en 3 etapas: 1) escoger los conceptos variables, 2) determinar los arcos que muestran las relaciones entre los conceptos y 3) asignar apropiadamente signos y la intensidad lingüística para describir las relaciones. Luego se debe elegir un método que permita simular el fenómeno, es decir, adoptar las herramientas adecuadas que permitan analizarlo y en lo posible, predecir el comportamiento ante nuevas situaciones. 
La Figura 4 relaciona dos conceptos A y B con un tercero C. Este es el modo de construir una simple relación causal entre dos conceptos A y B que influyen positiva o negativamente en otro concepto $\mathrm{C}$.

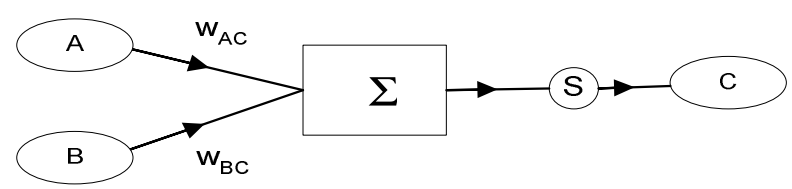

Figura 4: Red neuronal para inferir el valor de C

En este caso, puede considerarse que existe una relación causal entre los nodos a A-C y B-C, de manera que un incremento en el nodo A causa un incremento en el nodo $\mathrm{C}$ y a su vez, un incremento en el nodo B incrementa el valor del concepto $\mathrm{C}$ (si ambos pesos son positivos). Si se designan los pesos (intensidad) de estas conexiones con wAC y wBC respectivamente, el objeto del estudio consiste en implementar un procedimiento que permita establecer un nuevo valor de $\mathrm{C}$ y encontrar el estado de equilibrio mediante un proceso iterativo que garantice que al continuar el procedimiento los valores de todos los conceptos A, B y C, ya no sufrirán cambios. Es decir, se espera que después de un cierto número de iteraciones, el sistema se estabilice y converja a un estado fijo o bien a un ciclo de estados.

Como se mencionó, la técnica de los MCD no es infalible y puede ocurrir que no se llegue a un estado de equilibrio. Sin embargo, los MCD constituyen una herramienta muy flexible que permite incorporar o quitar nodos y volver a analizar la presencia de estabilidad en el proceso iterativo.

La metodología propia de la construcción y el procesamiento de un MCD se describe e ilustra a continuación mediante un problema concreto de formación estratégica presentado por Hilera y Martínez (2000) que puede ser tratado con un MCD. En el mismo se considerarán los siguientes conceptos que componen el MCD: Inversión Extranjera (IE), Leyes Regulatorias (LR), Empleo (E), Conflictividad Social (CS) y Estabilidad de Gobierno (EG). Las relaciones causales entre estos conceptos se ilustran en la Figura 5.

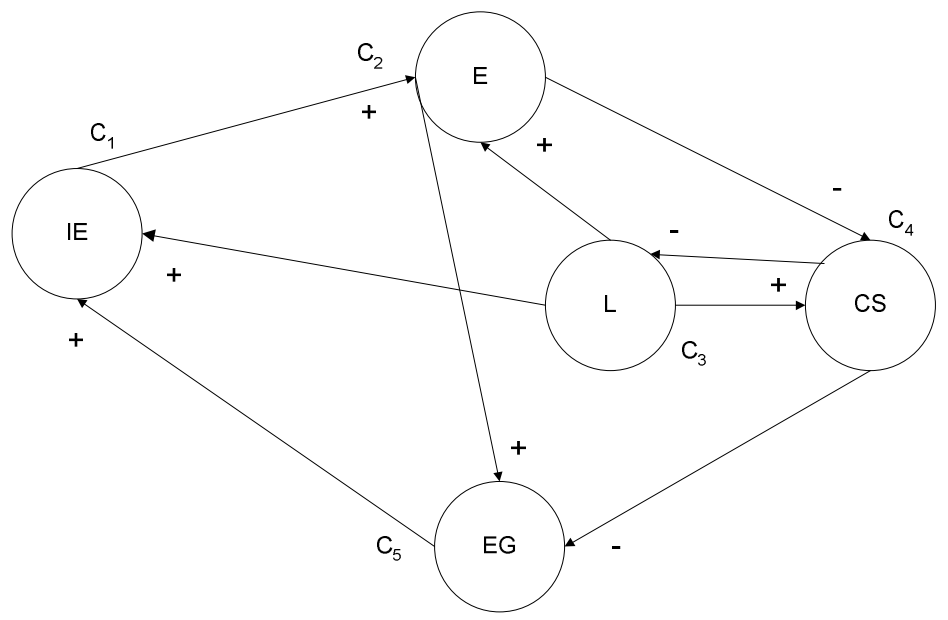

Figura 5: MCD para relacionar cinco conceptos 
Los MCD conllevan una técnica iterativa según la cual cada concepto va modificando su valor. En cada iteración se simula el paso correspondiente a un intervalo de tiempo determinado y el valor de cada concepto en la iteración actual es calculado a partir de los valores de sus conceptos antecedentes en la iteración anterior. Debido al carácter iterativo del procedimiento, el sistema representado por un MCD evoluciona a lo largo del tiempo hacia nuevos estados, es decir que, a partir de un estado inicial dado por un vector $\mathrm{C}$ cuyas componentes son los valores de los conceptos $\mathrm{C}=[\mathrm{IE}, \mathrm{E}, \mathrm{L}, \mathrm{CS}$, EG], se llega a un nuevo estado y a semejanza del sistema real podrá o no converger a un estado o ciclo de estados de equilibrio. De esta forma, en un MCD el valor de cada concepto representado en el mapa depende del nivel de sus antecedentes en la iteración anterior, y es calculado por medio de una suma de productos a la que se le aplica una función de salida. La relación entre un concepto y sus antecedentes es modelada por un arco que los vincula y que tiene asignado un peso que establece la intensidad de la influencia de un concepto sobre el otro. En este trabajo los nodos adoptarán sólo dos valores posibles, 0 ó 1 reservándose el término difuso para los valores de las aristas donde cada arco tiene asociado un peso cuyos valores se distribuyen en el intervalo [-1, $1]$.

\section{Procesamiento de un mapa cognitivo difuso}

La representación del nivel de causalidad entre conceptos queda mejor organizada si previamente se dispone de una tabla denominada tabla de causalidad o conectividad. La tabla 1 relaciona los conceptos de la Figura 4 mediante los pesos $w_{i j}$, cada valor representa la intensidad con la que un concepto i influye sobre otro concepto j.

\begin{tabular}{|l|l|l|l|l|l|}
\hline & $\mathrm{IE}$ & $\mathrm{E}$ & $\mathrm{L}$ & $\mathrm{CS}$ & $\mathrm{EG}$ \\
\hline $\mathrm{IE}$ & $w_{11}$ & $w_{12}$ & $w_{13}$ & $w_{14}$ & $w_{15}$ \\
\hline $\mathrm{E}$ & $w_{21}$ & $w_{22}$ & $w_{23}$ & $w_{24}$ & $w_{25}$ \\
\hline $\mathrm{L}$ & $w_{31}$ & $w_{32}$ & $w_{33}$ & $w_{34}$ & $w_{35}$ \\
\hline $\mathrm{CS}$ & $w_{41}$ & $w_{42}$ & $w_{43}$ & $w_{44}$ & $w_{45}$ \\
\hline $\mathrm{EG}$ & $w_{51}$ & $w_{52}$ & $w_{53}$ & $w_{54}$ & $w_{55}$ \\
\hline
\end{tabular}

Tabla 1: Nivel de causalidad en los conceptos de la Figura 4

A su vez, la representación tabular es llevada a una matriz para su procesamiento. Si por ejemplo, el concepto $\mathrm{C} 1$ se relaciona con el concepto $\mathrm{C} 2$ mediante el peso $w_{12}$ de valor 0.6, significa que la inversión Extranjera (IE) favorece el Empleo (E) con un factor de 0.6 en el sentido que un incremento en la inversión provoca un aumento en el empleo. La matriz completa se representa como sigue:

$$
w=\left(\begin{array}{lllll}
w_{11} & w_{12} & w_{13} & w_{14} & w_{15} \\
w_{21} & w_{22} & w_{23} & w_{24} & w_{25} \\
w_{31} & w_{32} & w_{33} & w_{34} & w_{35} \\
w_{41} & w_{42} & w_{43} & w_{44} & w_{45} \\
w_{51} & w_{52} & w_{53} & w_{54} & w_{55}
\end{array}\right)
$$


El procedimiento iterativo permite seguir la evolución del modelo en pasos que sucesivamente van entregando secuencias de estados con valores actualizados del vector de conceptos $\mathrm{C}$, este vector contiene todos los valores de los conceptos: $\mathrm{C}=[\mathrm{IE}, \mathrm{E}, \mathrm{L}$, $\mathrm{CS}, \mathrm{EG}$. Cada nuevo estado del vector $\mathrm{C}$ se obtiene aplicando la función $f$ al producto entre el vector $\mathrm{C}$ y la matriz de conectividad $w$. La obtención de los nuevos valores de $\mathrm{C}$ en el paso t+1 en función de los valores de $\mathrm{C}$ en el paso t está dada por la ecuación (2)

$$
C_{t+1}=f\left(C_{t} w\right)
$$

En esta expresión:

$C_{t}$ Es el vector con los valores de los conceptos en paso t.

$C_{t+1}$ Representa el vector que contiene todos los valores de los conceptos en la etapa $\mathrm{t}+1$ de la iteración.

$w$ Designa la matriz de conectividad

$f$ Es la función de transferencia que se aplica al producto $C_{t} w$

En el caso más simple $f$ es la función salto unitario que toma el valor cero si el argumento es menor que cero y valor uno si el argumento es mayor o igual a cero. La elección de esta función está ligada tanto a la simplicidad que se quiera tener durante el cálculo como a las características de convergencia que exhiba el método. En muchas ocasiones basta considerar la función salto unitario para el cálculo, mientras que para analizar la evolución de los conceptos a lo largo de las sucesivas iteraciones se recurre a la función identidad. Este último aspecto posibilita una característica de análisis adicional que tienen los MCD, ya que ir representando gráficamente los valores que van tomando los conceptos en el cálculo da una idea concreta de las oscilaciones y las situaciones por las que deberá pasar el sistema hasta llegar a un estado de equilibrio.

La matriz w tiene una importancia central en el procedimiento de construcción de un MCD. Dado que cada elemento de w contiene un peso que da la intensidad con que un concepto incide en otro (con tendencia a aumentarlo o disminuirlo), esta matriz con la conexión entre conceptos frecuentemente está construida con la ayuda de varios expertos en el área de interés y comúnmente se encuentran resultados muy disímiles entre especialistas al valorar un mismo hecho o situación, tales situaciones surgen cuando, por ejemplo, un médico debe establecer un diagnóstico basándose en los síntomas del paciente o cuando un juez debe aplicar una pena en base a los delitos cometidos y a la interpretación de las legislaciones vigentes.

En caso que se quiera considerar la opinión de varios expertos para construir la matriz de conectividad $\mathrm{w}$, habrá que incluir tantas matrices como expertos intervengan en el análisis y a partir de ahí obtener una nueva matriz de consenso resultante de operar con las matrices individuales basadas en la opinión de cada especialista. En este caso para determinar la matriz de pesos $\mathrm{w}$, que considere la opinión de todos los especialistas consultados sobre el tema, se recurre a la suma lógica difusa. El valor final del peso $\mathrm{w}_{\mathrm{ij}}$ que intervendrá en el estudio de la evolución de los conceptos surge de la ecuación (3)

$w_{i j}=\bigcup_{k=1}^{k=M} w_{i j}^{k}=\max \left(w_{i j}^{1}, w_{i j}^{2}, \ldots, w_{i j}^{M}\right)$

Donde se consideró la opinión de $\mathrm{M}$ expertos y por esa razón el supra índice de los pesos $w_{i j}$ recorre los valores de 1 a M. Durante el cálculo de la suma lógica borrosa 
para determinar la matriz de consenso $w$, se considera que cada peso ij se obtendrá como el de mayor valor absoluto para esos subíndices. Esta operación se realiza con los elementos correspondientes de las M matrices sugeridas por los expertos.

La lógica difusa proporciona muchas herramientas para tratar este tipo de problemas donde distintas personas tienen un grado de valoración particular sobre cada aspecto o situación determinada. Supongamos que se quiere determinar el valor correspondiente a $w_{12}$ (nivel de causalidad de C1 sobre C2). En este caso se trata de establecer cómo influye la inversión extranjera en el empleo. Un especialista puede establecer que tal influencia se da con un valor cercano a 1 , por ejemplo 0.9 , mientras que otro puede atribuirle un grado de influencia de 0.7. Procediendo de acuerdo con la ecuación (3), para determinar el valor $w_{12}$ se debe adoptar el máximo entre los dos valores que proponen los especialistas. La regla del máximo es una de las operaciones más simples de la lógica difusa pero proporciona una solución al problema de hallar la matriz de conectividad $w$. En la bibliografía se citan trabajos donde aparecen formulaciones con mayor énfasis en la aplicación de reglas difusas para construir la matriz de adyacencia de un MCD (Hilera \& Martínez, 2000; Carvalho \& Tomé, 1999)

\section{RESULTADOS}

La estrategia seguida para la construcción de un MCD proporciona un camino para obtener la evolución que siguen los conceptos a partir de un estado inicial determinado. A continuación se mostrarán los resultados del análisis del problema que relaciona los cinco conceptos seleccionados para establecer relaciones causales entre ellos a través de un MCD, representado gráficamente en la Figura 4. Estos conceptos están dados por el vector $\mathrm{C}=[\mathrm{IE}, \mathrm{E}, \mathrm{L}, \mathrm{CS}, \mathrm{EG}]$. Si se considera que la opinión de los dos expertos ha sido reunida en las matrices $w_{1}$ y $w_{2}$, la matriz de adyacencia resultante al aplicar el operador U de unión que, como ya se mencionó, consiste en tomar el mayor valor de los dos que corresponden a una misma posición de la matriz de cada especialista, es la siguiente:

$$
\begin{gathered}
w_{1}=\left(\begin{array}{ccccc}
0 & 0.6 & 0 & 0 & 0 \\
0 & 0 & 0 & -1 & 0.2 \\
0.2 & 0.4 & 0 & 0.6 & 0 \\
0 & 0 & 0 & 0 & -0.8 \\
0.7 & 0 & 0 & 0 & 0
\end{array}\right) \\
w_{2}=\left(\begin{array}{ccccc}
0 & 0.3 & 0 & 0 & 0 \\
0 & 0 & 0 & -0.2 & 0.2 \\
0.8 & 0.1 & 0 & 0.3 & 0 \\
0 & 0 & -0.2 & 0 & -0.6 \\
1 & 0 & 0 & 0 & 0
\end{array}\right) \\
w=w_{1} \bigcup w_{2}=\left(\begin{array}{ccccc}
0 & 0.6 & 0 & 0 & 0 \\
0 & 0 & 0 & -1 & 0.2 \\
0.8 & 0.4 & 0 & 0.6 & 0 \\
0 & 0 & -0.2 & 0 & -0.8 \\
1 & 0 & 0 & 0 & 0
\end{array}\right)
\end{gathered}
$$

Esta matriz w se mantiene constante a lo largo de todo el proceso iterativo que conlleva la evolución que van sufriendo los conceptos en cada una de las iteraciones. 
Inicialmente se parte de un estado inicial con el que se fijan los valores de los cinco conceptos según el vector $\mathrm{C}(\mathrm{t}=0)=[0,0,0,1,0]$.

$\mathrm{El}$ primer paso consiste en efectuar el producto entre el vector fila $\mathrm{C}$ y la matriz $w$, obteniéndose como resultado el vector $\mathrm{R}=\left[\begin{array}{lllll}0 & 0.6 & -0.2 & 0 & -0.8\end{array}\right]$.

De acuerdo con la ecuación 2, el siguiente paso consiste en aplicarle la función $\mathrm{f}$ a este valor obtenido. En este trabajo se adoptará una función salto unitario para el cálculo iterativo y la función identidad para seguir la evolución de los conceptos (Carlsson et al., 2006; Peláez et al., 1995).

La Tabla 2 contiene los valores que se van obteniendo para los sucesivos pasos de $\mathrm{t}$, desde 0 a 3 . En la parte inferior derecha puede observarse cómo las dos últimas líneas han permanecido iguales. Esto significa que los valores de $\mathrm{C}$ para $\mathrm{t}=2$ son iguales a los correspondientes a $\mathrm{t}=3$, es decir, el sistema ha llegado a un estado de equilibrio a partir del cual no sufrirá variaciones. El resto de los cálculos parciales aparecen detallados en la tabla. Esta situación puede interpretarse de la siguiente manera: si se parte del estado inicial donde $\mathrm{C}(\mathrm{t}=0)=[0,0,0,1,0]$ luego de tres iteraciones, se llega a una estabilidad en los valores de los conceptos que componen el mapa, es decir, $\mathrm{C}(\mathrm{t}=3)$ $=\mathrm{C}(\mathrm{t}=4)=[1,1,1,0,1]$. Por lo tanto, partiendo de una situación inicial $\mathrm{C}(\mathrm{t}=0)$ de plena conflictividad social se llega a un estado $\mathrm{C}(\mathrm{t}=5)$ donde ésta se ha podido eliminar con Inversión Extranjera (IE), aumentando el Empleo (E) y en un marco de Leyes Regulatorias (L), logrando además la Estabilidad en el Gobierno (EG).

\begin{tabular}{|c|c|c|c|}
\hline $\mathrm{t}$ & $\mathrm{C}(\mathrm{t})$ & $\mathrm{R}=\mathrm{C}^{*} \mathrm{w}$ & $\mathrm{C}(\mathrm{t}+1)=\mathrm{f}(\mathrm{R})$ \\
\hline 0 & {$[0,0,0,1,0]$} & {$[0,0.6,-0.2,0,-0.8]$} & {$[1,1,0,1,0]$} \\
\hline 1 & {$[1,1,0,1,0]$} & {$[0,0.6,-0.2,-1,-0.6]$} & {$[1,1,0,0,0]$} \\
\hline 2 & {$[1,1,0,0,0]$} & {$[0,0.6,0,-1,0.2]$} & {$[1,1,1,0,1]$} \\
\hline 3 & {$[1,1,1,0,1]$} & {$[1.8,1,0,-0.4,0.2]$} & {$[1,1,1,0,1]$} \\
\hline
\end{tabular}

Tabla 2: Valores de los conceptos durante la evolución del MCD

La Figura 6 representa la evolución de los conceptos durante el proceso iterativo. 

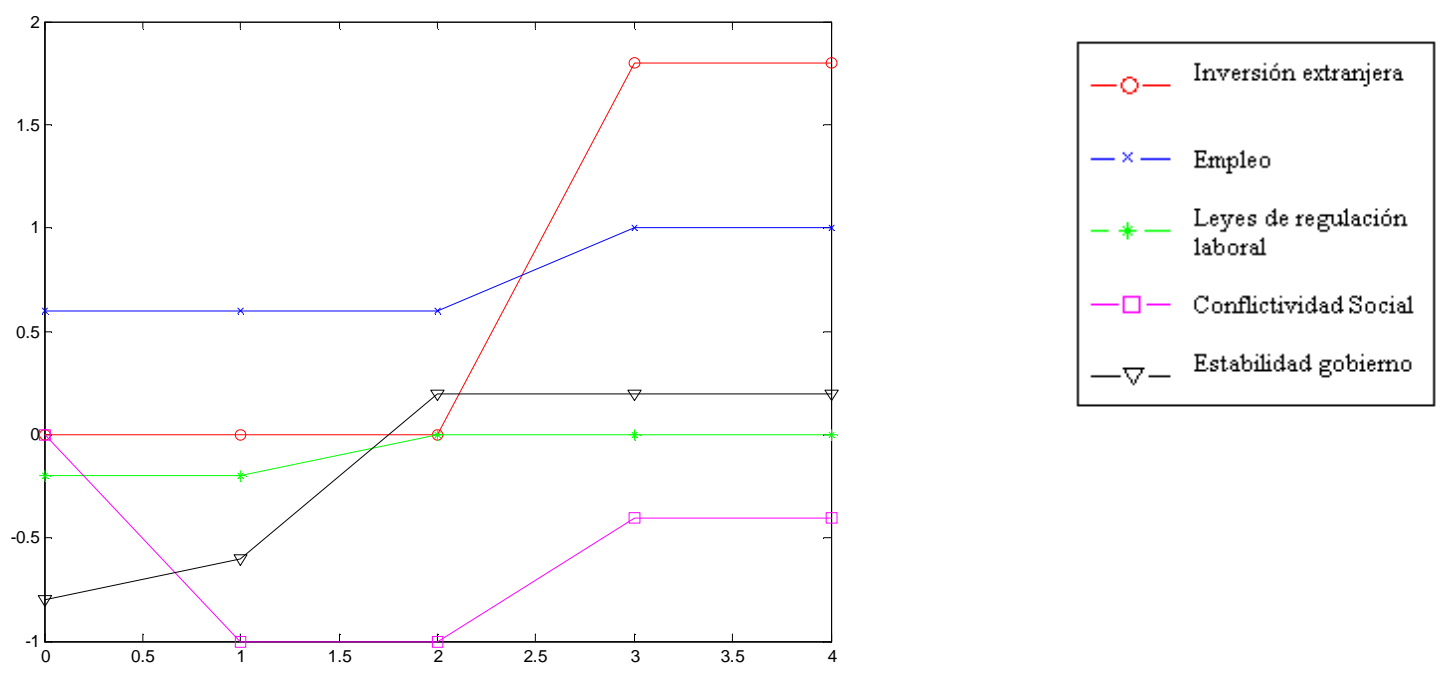

Figura 6: Oscilaciones de los conceptos durante la evolución del MCD

Por otro lado, si se parte del estado inicial donde $\mathrm{C}(\mathrm{t}=0)=[0,0,1,1,0]$ luego de tres iteraciones, nuevamente se llega a una estabilidad en los valores de los conceptos.

\begin{tabular}{|c|c|c|c|}
\hline $\mathrm{t}$ & $\mathrm{C}(\mathrm{t})$ & $\mathrm{R}=\mathrm{C}^{*} \mathrm{w}$ & $\mathrm{C}(\mathrm{t}+1)=\mathrm{f}(\mathrm{R})$ \\
\hline 0 & {$[0,0,1,1,0]$} & {$[0.8,0.4,-0.2,0.6,-0.8]$} & {$[1,1,0,1,0]$} \\
\hline 1 & {$[1,1,0,1,0]$} & {$[0,0.6,-0.2,-1,-0.6]$} & {$[1,1,0,0,0]$} \\
\hline 2 & {$[1,1,0,0,0]$} & {$[0,0.6,0,-1,0.2]$} & {$[1,1,1,0,1]$} \\
\hline 3 & {$[1,1,1,0,1]$} & {$[1.8,1,0,-0.4,0.2]$} & {$[1,1,1,0,1]$} \\
\hline
\end{tabular}

Tabla 3: Valores de los conceptos durante la evolución del MCD

En la Tabla 3 se observa el proceso iterativo y en la Figura 7 la evolución de los valores de los mismos en dicho proceso.
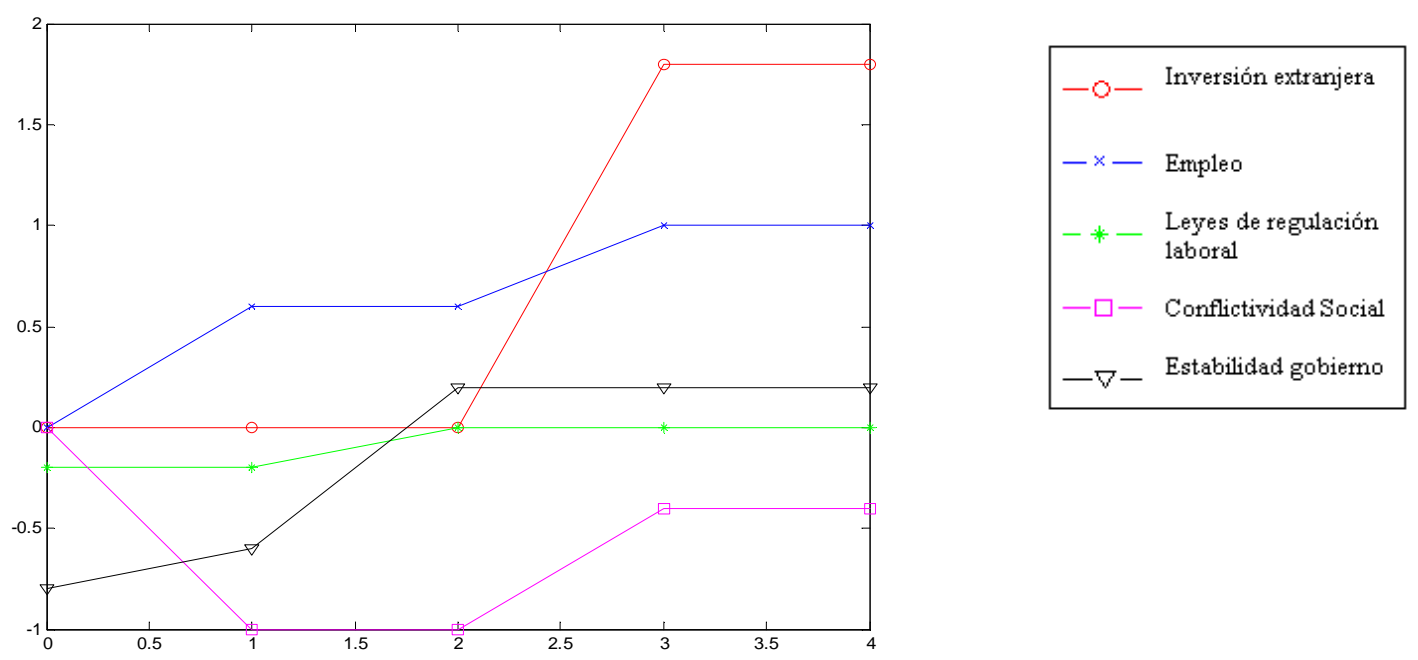

Figura 7: Oscilaciones de los conceptos correspondientes a la tabla 2 
En este caso, se parte de una situación inicial de plena conflictividad social pero con un marco de leyes laborales. Luego de las iteraciones se llega a un estado $\mathrm{C}(\mathrm{t}=5)$ donde se observa que para eliminar la Conflictividad Social y garantizar la Estabilidad en el Gobierno (EG) es necesario contar con Inversión Extranjera (IE) que aumente el Empleo (E).

A partir del análisis de los resultados obtenidos en los dos casos concretos, se observa que el MCD construido proporciona resultados claros y fácilmente interpretables. Dado que el número de conceptos es bajo, no se hace necesario recurrir a otras técnicas complementarias como se propone en los trabajos de Stach et al. (2010) y Kannappan et al. (2011). En consecuencia es importante observar que estos resultados fueron obtenidos únicamente a partir de la construcción de la matriz de adyacencia mediante la opinión de expertos altamente calificados en la problemática concreta que se propuso analizar y que en ningún momento fue necesario recurrir a otras técnicas adicionales para optimizar el procedimiento de construcción de un MCD.

\section{CONCLUSIONES}

Los MCD, al igual que otros mapas como los conceptuales y los mapas cognitivos tradicionales, sirven para representar el conocimiento mediante conceptos unidos por nexos. Sin embargo, los MCD tienen características adicionales que permiten detectar el conocimiento implícito sobre situaciones causales y la evolución de las mismas cuando se parte de determinadas situaciones externas que quedan reflejadas en los valores de los conceptos y en los valores con que las relaciones causales influyen sobre ellos. En este trabajo se muestra el procedimiento iterativo que constituye el núcleo operativo de un MCD y la forma en que esta técnica permite seguir la dinámica de los conceptos reunidos en el mapa. Mediante un ejemplo concreto del campo de la economía se pone de manifiesto la sensibilidad que presenta la evolución del mapa, cuando partiendo de una situación inicial determinada es posible analizar los sucesivos estados por los que pasan los conceptos, brindando además la posibilidad de interpretar estos paulatinos cambios como estados que experimentará el sistema en el contexto real en el que se está estudiando el problema. Los MCD muestran ser una herramienta de aplicación directa y eficiente ya que ofrece una forma rápida y de fácil empleo como para constituir un soporte ideal destinado al especialista de un área temática que quiere investigar un problema concreto y disponer de un curso de acción a seguir. La técnica de los MCD permite seguir con comodidad la evolución que presentan los conceptos reunidos en el mapa preservando un gran impacto visual y uniendo técnicas de análisis cuantitativo a fenómenos que muchas veces son sólo descriptos en términos cualitativos. Del análisis realizado se desprende que es recomendable mantener un diseño sencillo del MCD en los casos en que el número de conceptos sea bajo y anexarle a la construcción de un MCD clásico algoritmos de depuración más específicos sólo en los casos en que haya gran cantidad de conceptos involucrados en la problemática que se analiza.

Las líneas de investigación en lo que se refiere a los mapas cognitivos difusos están dadas en una dirección tendiente a integrar las técnicas de lógica difusa conjuntamente con las otras dos ramas de la soft computing: las redes neuronales y los algoritmos genéticos. De particular interés resulta el caso en el que debido a la opinión de especialistas la información es integrada a través de un gran número de conceptos 
que muchas veces conforman pensamientos o puntos de vista que resultan contradictorios o entran en conflicto entre sí. En estos casos es útil recurrir a métodos de decisión multicriterio en los que básicamente el planteo consiste en utilizar mapas cognitivos y técnicas de lógica difusa para ayudar a grupos de personas a tomar una decisión cuando existen varias aristas desde donde el problema puede ser analizado (Rodriguez Bello, 2009). Si bien en este trabajo no se emplean algoritmos genéticos ni redes neuronales puede resultar de interés, como futura línea de investigación, buscar soluciones a estos problemas y analizar los resultados que se obtienen con la incorporación de dichas técnicas de la inteligencia artificial, comparándolos con rutinas de solución más básicas.

\section{REFERENCIAS}

Axlerod, R. (1976). Structure of Decision: the Cognitive Maps of Political Elites. Princeton University Press, Princeton, New Jersey.

Cai, Y., Miao, Ch., Tan, A. \& Shen Z. (2006). Context Modeling with Evolutionary Fuzzy Cognitive Map in Interactive Storytelling. School of Computer Engineering, Nanyang Technological University.

Calais, G. (2008). Fuzzy Cognitive Maps Theory: Implications for Interdisciplinary Reading: National Implications. FOCUS On Colleges, Universities, and Schools, 2(1), $1-16$.

Carlsson, C. (1996). Knowledge formation in strategic management, HICSS-27 Proceedings, IEEE Computer Society Press, Los Alamitos.

Carlsson, C., Ramírez, L., Mora, M. \& Terán, A. (2006). Adaptive Fuzzy Cognitive Maps for Hyperknowledge Representation in Strategy Formation Process. Proceedings of the International Panel Conference on Soft and Intelligent Computing, Technical Univ. of Budapes. http://citeseer.ist.psu.edu.

Carvalho,J.P. \& Tomé, J.A. (1999). Rule Based Fuzzy Cognitive Maps and Fuzzy Cognitive Maps - A Comparative Study , Proceedings of the 18th International Conference of the North American Fuzzy Information Processing Society, NAFIPS99, New York.

Chandana, S., Leung, H. \& Levy, J. (2007). Disaster management model based on Modified Fuzzy Cognitive Maps. Systems, Man and Cybernetics. ISIC. IEEE International Conference.

Hilera, J. R. \& Martínez V. (2000). Redes Neuronales Artificiales: Fundamentos, modelos y aplicaciones. RA-MA Editorial, Madrid.

Jang, R. Sun, C. \& Mitsutani, E. (1997). Neuro-Fuzzy and Soft Computing, A Computational Approach to Learning and Machine Intelligence. Nueva Jersey, Prentice Hall.

Kannappan, A., Tamilarasi, A. \& Papageorgiou, E. (2011). Analyzing the performance of fuzzy cognitive maps with non-linear hebbian learning algorithm in predicting autistic disorder. Expert Systems with Applications: An International Journal archive. Volume 38 Issue 3. 
Kardaras, B. \& Karakostas B. (1999). The use of fuzzy cognitive maps to simulate the information systems strategic planning process. Information and Software Technology, 41, 197-210.

Kosko, B. (1986) Fuzzy Cognitive Maps. International Journal on Man Machine Studies. 24. 65-75.

Kosko, B. (1997). Fuzzy Engineering. Prentice-Hall, Upper Saddle River, New Jersey.

Lin, Ch., Lu P., Wu W., Chen Ch., Chiang H. \& Huang, Y. (2010). Utilizing a concept map as the teaching strategy based on conceptual change theory for the course information technology and society. Joint International IGIP-SEFI Annual Conference 2010, 19th - 22nd September 2010, Trnava, Slovakia.

Mellado, V., Peme-Aranega, C., Redondo, C. \& Bermejo, M. L. (2002). Los mapas cognitivos en el análisis gráfico de las concepciones del profesorado. Campo Abierto, $22,37-58$.

Miao, Ch., Yang, Q., Fang, H. \& Goh, A. (2006). A cognitive approach for agent-based personalized recommendation. Knowledge-Based Systems, 20, 397-405.

Novak, J.D. \& Gowin, D.B. (1988). Aprendiendo a aprender. Barcelona: Martínez Roca.

Papageorgiou, E., Stylios, Ch. \& Groumpos P. (2006). Unsupervised learning techniques for fine- tuning fuzzy cognitive map causal links, Intl. Journal of HumanComputer Studies. 64, 727-743.

Papageorgiou, E. Markinos, A. \& Gemtos, T. (2011). Fuzzy cognitive map based approach for predicting yield in cotton crop production as a basis for decision support system in precision agriculture application. Applied Soft Computing, Volume 11 Issue 4

Peláez, C. E. \& Bowles, J. B. (1995). Applying Fuzzy Cognitive Maps KnowledgeRepresentation to Failure Modes Effects Analysis. IEEE Proceedings Annual Reliability and Maintainability Symposium. 0149-144X/95.

Pimienta, J. (2005). Metodología constructivista: Guía para la planeación docente. Pearson/Prentice-Hall. http://slideshare.net/eus/mapas-cognitivos-y-construccin-delconocimiento

Rodriguez Bello, S. (2008). Toma de decisión multi-criteria con AHP, ANP y Lógica Difusa. Maestría en Ingeniería de Sistemas y Computación. Universidad Nacional de Colombia.

Stach, W., Kurgan, L. Pedrycs, W. \& Reformat, M. (2005). Genetic learning of fuzzy cognitive maps. Fuzzy Sets and Systems. 153(3), 371-401.

Stach, W., Kurgan, L. \& Pedrycz, W. (2010). A divide and conquer method for learning large Fuzzy Cognitive Maps. Journal Fuzzy Sets and Systems archive. Vol. 161 Issue 19

Stylios, Ch., Georgopoulos, V. \& Groumpos, P. (2005). The use of fuzzy cognitive maps in modeling systems. School of Electrical Engineering and Computer Science, Ohio University.

Wheeldon, J. \& Faubert J. (2009). Framing Experience: Concept Maps, Mind Maps, and Data Collection in Qualitative Research. Int 10. 